\title{
AHLFORS AND CONFORMAL INVARIANTS
}

\author{
Albert Baernstein II*
}

\section{Introduction}

Conformal invariants include quantities such as harmonic measures, hyperbolic distances and extremal lengths which do not change under conformal mapping. It would be quite impossible to give a systematic introduction in a small space, or even to discuss most of Ahlfors's contributions to this subject. Thus, this article is in the nature of an excursion. Our point of departure will be the conjecture of Denjoy about asymptotic values of entire functions, which Ahlfors proved in 1928 by means of his "distortion theorem". We shall examine some work leading up to this proof, and the proofs of the conjecture itself by Ahlfors and by Carleman and Beurling which came soon after that of Ahlfors. Each of the three proofs spawned theories and problems which are still of great interest in complex function theory and beyond, and we shall follow some of these threads down to the present day.

Perhaps the main theme of the subject is to find relations between conformal and Euclidean quantities. During our journey, we shall visit some problems that are now essentially settled, such as the relation between harmonic and Hausdorff measure in a simply connected domain (Section 6), and others that are not, notably Painlevé's problem of geometrically characterizing null sets for bounded analytic functions (Section 7). For the "angular derivative problem" (Section 5), the authorities differ as to whether the problem is or is not completely solved. These subjects have all been strongly influenced by the work of Ahlfors, either directly, or indirectly through tools such as extremal length in whose development he has played a major role.

My choice of topics has been somewhat arbitrary. I regret especially having no space to discuss hyperbolic metrics or extremal lengths for multiple curve families. See [Mi], [We 2], and [I] for some intresting recent results. Also, I would have liked to discuss work related to Bloch's theorem, but there is time only to note that the Ahlfors-Grunsky conjecture of 1937 about the exact value of Bloch's constant has still been neither proved nor disproved.

\footnotetext{
* This research was supported by a grant from the National Science Foundation.
} 


\section{The Denjoy conjecture}

An entire function $f$ is said to have the asymptotic value $b$ if there is a path $\gamma$ in the plane $\mathbf{C}$ such that $f(z) \rightarrow b$ as $z \rightarrow \infty$ along $\gamma$. Denjoy conjectured in 1907 that if $f$ has $n$ different finite asymptotic values then its order is at least $n / 2$. That is, its maximum modulus $M(r)$ on $|z|=r$ must satisfy

$$
\limsup _{r \rightarrow \infty} \frac{\log \log M(r)}{\log r} \geq \frac{1}{2} n .
$$

He states that he can prove his conjecture when the asymptotic paths are rays from the origin. Simple examples show that for each positive integer $n$ entire functions of order $n / 2$ exist with $n$ different asymptotic values.

If $f$ has $n$ asymptotic values $b_{i}$ there are corresponding smooth paths $\gamma_{i}$ which begin at the origin but are otherwise disjoint. These paths divide the plane into $n$ simply connected domains $\Omega_{i}$, and $f$ is bounded on each $\partial \Omega_{i}$, say $|f| \leq 1$ there.

If we assume the $b_{i}$ are distinct, it follows from a theorem of Lindelöf from 1908 that $f$ must be unbounded in each $\Omega_{i}$. Assume that the $\Omega_{i}$ are in fact angular sectors, $\Omega_{i}=\left\{z:\left|\arg z-\varphi_{i}\right|<\beta_{i}\right\}$. F. and R. Nevanlinna in 1922 , as an application of their "two-constant" theorem, had proved a sharp

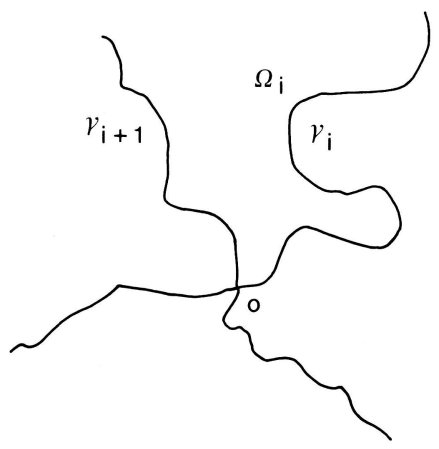
form of the Phragmén-Lindelöf principle: If $f$ is holomorphic in a sector $|\arg z|<$ $\beta$ and bounded on the sides, then if $f$ is unbounded inside its maximum modulus must grow quickly,

$$
\liminf _{r \rightarrow \infty} r^{-\alpha} \log M(r)>0,
$$

where $\alpha=\pi / 2 \beta$. At least one of the $\beta_{i}$ is $\leq \pi / n$. Thus, the maximum modulus of $f$ on $|z|=r$ must satisfy

$$
\liminf _{r \rightarrow \infty} r^{-n / 2} \log M(r)>0,
$$

which proves Denjoy's conjecture, in the sector case, in a stronger form.

To extend this argument to the case of general $\Omega_{i}$, one needs a theorem of Phragmén-Lindelöf type for "curvilinear sectors". A result of this sort was found by Carleman in 1920. Under the mapping $w=\log z$ the $\Omega_{i}$ become striplike domains, which we will also call $\Omega_{i}$. For any domain $\Omega$, Carleman defines

$$
\Theta(x)=\text { total length of } \Omega \cap\{z: \operatorname{Re} z=x\} .
$$

Suppose that $f$ is analytic in $\Omega$, and define

$$
M(x, f)=\sup \{|f(z)|: z \in \Omega, \operatorname{Re} z=x\} .
$$


Carleman assumes that $\Theta(x)<\infty$ for every $x$ and that $|f| \leq 1$ on $\partial \Omega$. If $f$ is not bounded in $\Omega$ then $M(x)>1$ for $x>x_{0}$. Carleman proves a first order differential inequality for $M(x)$ :

$$
\frac{d}{d x} \log \log M(x) \geq \frac{4}{\pi \Theta(x)}, \quad x>x_{0} .
$$

According to Beurling [Be] the proof is the first application of the method of harmonic majorization. Integration shows that, when $f$ is unbounded and $x_{0}<x_{1}<x_{2}$,

$$
\frac{4}{\pi} \int_{x_{1}}^{x_{2}} \frac{d x}{\Theta(x)} \leq \log \log M\left(x_{2}\right)-\log \log M\left(x_{1}\right) .
$$

The use of integrals of type $\int \Theta^{-1}(x) d x$ to measure the size of a domain apparently originated with Carleman. It is surely one of the most brilliant ideas in the history of complex function theory.

Returning to the situation of Denjoy's conjecture, let $\Theta_{i}(x)=\Theta\left(x, \Omega_{i}\right)$. Then $\sum_{i=1}^{n} \Theta_{i}(x) \leq 2 \pi$, and hence

$$
n^{2} \leq 2 \pi \sum_{i=1}^{n} \frac{1}{\Theta_{i}(x)} .
$$

For $x>x_{1}>x_{0},(1.2)$ and (1.3) imply

$$
n\left(x-x_{1}\right) \leq \frac{\pi^{2}}{2}\left(\log \log M(x)-C\left(x_{1}\right)\right) .
$$

For the original entire function $f$, this gives (1.1) with $2 n / \pi^{2}$ instead of $n / 2$. Thus, Carleman had proved that an entire function of order $\varrho$ can have at most $\pi^{2} \varrho / 2$ asymptotic values.

To obtain the full Denjoy conjecture, the $4 / \pi$ in (1.2) must be replaced by $\pi$. Ahlfors discovered how to do this while in Zürich in 1928. His general idea is to conformally map the striplike domain $\Omega$ onto the rectilinear strip

$$
S=\left\{z:|\operatorname{Im} z|<\frac{1}{2}\right\}
$$

by an appropriate mapping $\Phi, \Phi: \Omega \rightarrow S$, and then apply the known PhragménLindelöf result to $f \circ \Phi^{-1}$. For such an argument to work one must have good control over the distortion of $\Phi$, and this is the content of what is now called Ahlfors's distortion theorem.

We follow the presentation in Ahlfors's thesis [A 1]. Assume that the simply connected domain $\Omega$ is unbounded on both the left and right. That is, $\Omega$ contains a 
curve $z=\beta(t), 0<t<1$, for which $\lim _{t \rightarrow 0} \operatorname{Re} \beta(t)=-\infty, \lim _{t \rightarrow 1} \operatorname{Re} \beta(t)=\infty$. Assume that $\operatorname{Re} \Phi(\beta(t)) \rightarrow-\infty$ as $t \rightarrow 0, \operatorname{Re} \Phi(\beta(t)) \rightarrow \infty$ as $t \rightarrow 1$. For each $x, \Omega \cap\{\operatorname{Re} z=x\}$ consists of one or more open intervals, at least one of which is a crosscut of $\Omega$ separating the prime ends at $-\infty$ and $\infty$ determined by the curve $\beta$. Let $\theta_{x}$ denote the crosscut which is first encountered by $\beta$. Assume that $\theta_{x}$ has finite length for every $x$, and define

$$
\theta(x)=\text { length } \theta_{x}, \quad u_{2}(x)=\sup _{\theta_{x}} \operatorname{Re} \Phi, \quad u_{1}(x)=\inf _{\theta_{x}} \operatorname{Re} \Phi .
$$
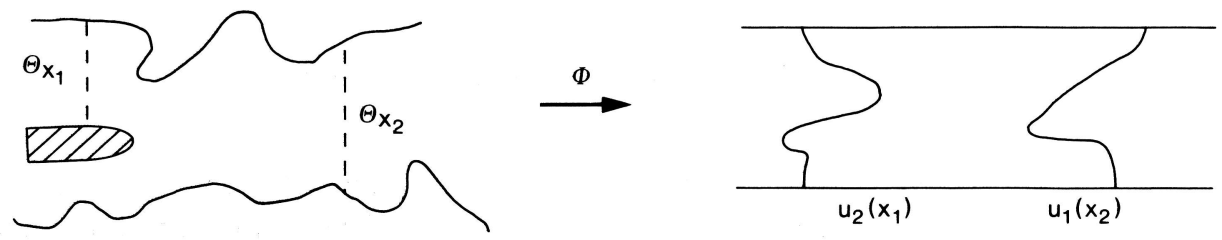

Ahlfors distortion theorem. If $\int_{x_{1}}^{x_{2}} \theta^{-1}(x) d x>2$ then

$$
\int_{x_{1}}^{x_{2}} \frac{d x}{\theta(x)} \leq u_{1}\left(x_{2}\right)-u_{2}\left(x_{1}\right)+4 \text {. }
$$

If $f$ is analytic in $\Omega$, bounded on $\partial \Omega$ and unbounded at $+\infty$, then for $g=f \circ \Phi^{-1}$ the Nevanlinna brothers' form of the PL principle gives

$$
\lim _{x \rightarrow+\infty} e^{-\pi x} \log M(x, g)>0 .
$$

A simple argument with the maximum principle leads to an inequality

$$
\log \log M(x, f) \geq \pi u_{1}(x)-O(1), \quad x \text { large. }
$$

For the domains which appear in the Denjoy conjecture we have $\theta(x) \leq$ $\Theta(x) \leq 2 \pi$. The distortion theorem is applicable when $x_{2}-x_{1}>4 \pi$. For sufficiently large $x_{1}, x_{2}$, we conclude that

$$
\pi \int_{x_{1}}^{x_{2}} \frac{d x}{\theta(x)} \leq \log \log M\left(x_{2}, f\right)+C
$$

where $x_{2}>x_{1}$ and $C$ depends only on $x_{1}$. Since $\theta(x) \leq \Theta(x)$, this gives a sharp version of (1.2), and the complete Denjoy conjecture follows, in the strong form (1.1).

The distortion theorem is one of the seminal results of our subject. We shall discuss it further in Section 4. In the next two sections we describe two other proofs of the Denjoy conjecture, by Carleman [Cmn] and Beurling [Be], which were published in 1933, both of which likewise have had strong influence on future developments. 


\section{Carleman's proof}

Carleman's new idea is to replace his nonsharp first order differential inequality for $M(x)$ by a sharp second order one for a certain quadratic mean. Assume again that $f$ is analytic in $\Omega,|f| \leq 1$ on $\partial \Omega$. The simple connectivity assumption on $\Omega$ is dropped. Define

$$
\theta^{*}(x)=\text { length of the longest arc in } \Omega \cap\{\operatorname{Re} z=x\},
$$

and also $Q(x)$ by

$$
Q^{2}(x)=\int_{-\infty}^{\infty}\left(\log ^{+}|f(x+i y)|\right)^{2} d y
$$

where $|f|$ is set equal to one for $x+i y \notin \Omega$. Carleman proves a convexity inequality for $Q(x)$,

$$
Q^{\prime \prime}(x) \geq \frac{\pi^{2}}{\theta^{*}(x)^{2}} Q(x) .
$$

The source of this inequality is the inequality of Wirtinger: If $h(y)$ is a piecewise smooth nonconstant function on $[a, b]$ with $h(a)=h(b)=0$, then

$$
\int_{a}^{b}\left(h^{\prime}(y)\right)^{2} d y \geq \frac{\pi^{2}}{(b-a)^{2}} \int_{a}^{b} h^{2}(y) d y
$$

with equality for $h(y)=\sin [\pi(y-a) /(b-a)]$.

Set $\varphi(x)=\log Q(x)$ and assume that $f$ is unbounded as $\operatorname{Re} z \rightarrow+\infty$. The convexity inequality can be integrated to give

$$
\pi \int_{x_{1}}^{x_{2}} \frac{d x}{\theta^{*}(x)} \leq \varphi\left(x_{2}\right)+C_{1} \varphi^{\prime}\left(x_{2}\right)+C_{2},
$$

when $x_{1}, x_{2}$ are large, and $C_{1}, C_{2}$ depend on $x_{1}$. In the Denjoy situation $\theta^{*}(x) \leq \Theta(x) \leq 2 \pi, Q(x) \leq(2 \pi)^{1 / 2} \log ^{+} M(x)$. A second integration of (2.1) to get rid of the term $\varphi^{\prime}$ and use of (1.3) then leads to a proof that an entire function with $n$ different asymptotic values indeed has order at least $n / 2$.

For just a moment let us think of our $\Omega$, which is not necessarily simply connected, as being sector-like instead of striplike. Define $\theta^{*}(r)=\infty$ when $\Omega$ contains the whole circle $|z|=r$. Otherwise, $\theta^{*}(r)$ will denote the angular measure of the longest arc in $\Omega \cap(|z|=r)$. Assume that $u$ is non-negative and subharmonic in $\Omega, u=0$ on $\partial \Omega$, and set $u=0$ outside $\Omega$. Tsuji [T, p. 116] observes that Carleman's analysis is still valid, and obtains for

$$
Q(r)^{2}=\frac{1}{2 \pi} \int_{0}^{2 \pi} u^{2}\left(r e^{i \varphi}\right) d \varphi
$$


the differential inequality

$$
\frac{d^{2} Q}{d(\log r)^{2}}(r) \geq \frac{\pi^{2}}{\theta^{*}(r)^{2}} Q(r) .
$$

Suppose that $\Omega$ meets the circle $|z|=R$. Apply this with

$$
u(z)=\omega_{R}(z)=\omega\left(z,|z|=R, \Omega_{R}\right),
$$

where $\Omega_{R}$ is a component of $\Omega \cap(|z|<R)$, and $\omega$ denotes harmonic measure. Tsuji cleverly integrates the convexity inequality to obtain a fundamental estimate for $\omega_{R}$. We state the version of [HWe, p. 123].

Tsuji's inequality. For each $\lambda \in(0,1)$, and $|a|<\frac{1}{2} \lambda R$,

$$
\omega_{R}(a) \leq 9(1-\lambda)^{-1 / 2} \exp \left(-\pi \int_{2|a|}^{\lambda R} \frac{d r}{r \theta^{*}(r)}\right) .
$$

Taking $\Omega$ to be a sector, one sees that the $\pi$ inside

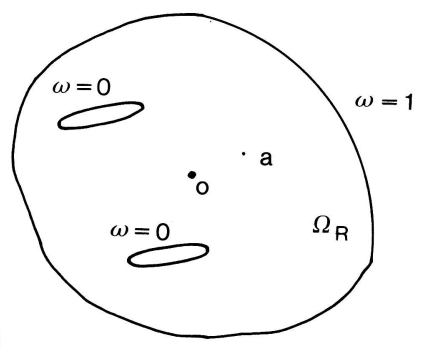
the exponent cannot be replaced with anything larger. When $\Omega$ is simply connected results of this type can be deduced from the distortion theorem or other arguments which involve conformal mapping, but for domains of arbitrary connectivity it appears that differential inequalities of Carleman type are one of the few known ways to achieve sharp estimates for harmonic measure.

To indicate just a sample of the many different situations in which Tsuji's inequality has been applied, we mention Weitsman's theorem [We 1] that $\sum_{a} \delta^{1 / 3}(a, f)<\infty$ for meromorphic functions of finite order, the work of Hayman and Weitsman [HWe] about means and coefficients of functions in the unit disk, and the recent work by Rossi $[R]$ and Shen $[\mathrm{S}]$ about zeros of solutions of differential equations $w^{\prime \prime}(z)+A(z) w(z)=0$, where $A$ is entire.

\section{Beurling's proof}

Let us return to the context of simply connected $\Omega$, which we again think of as being striplike, though possibly bounded. Beurling's proof [Be p. 104] of Denjoy's conjecture is notable, among other reasons, for its explicit use of harmonic measure in majorization arguments. Beurling notes that R. Nevanlinna had introduced the harmonic measure $\omega(z, E, \Omega)$ when $E$ is an arc in $\partial \Omega$, and that he, Beurling, proposes to consider it for more general boundary sets $E$. 
For $z_{0} \in \Omega, E \subset \partial \Omega$, Beurling defines another conformally invariant quantity, $\lambda$, which he calls "distance extrémale", by

$$
\begin{gathered}
\ell^{2}\left(z_{0}, E, \Omega\right)=\pi \frac{\inf (\text { length } \gamma)^{2}}{\operatorname{area}(\Omega)} \\
\lambda^{2}\left(z_{0}, E, \Omega\right)=\sup \ell^{2}\left(F\left(z_{0}\right), F(E), F(\Omega)\right)
\end{gathered}
$$

where the infimum is taken over all paths $\gamma$ in $\Omega$ which start at $z_{0}$ and end at a point of $E$, and the supremum is taken over all conformal mappings $F$ of $\Omega$ for which $F(\Omega)$ has finite area.

Beurling proves that the harmonic measure and distance extrémale are related by the inequality

$$
\omega\left(z_{0}, E, \Omega\right) \leq \exp \left(1-\lambda^{2}\left(z_{0}, E, \Omega\right)\right) .
$$

If $x_{1}<x_{2}, z_{0}=x_{1}+i y$, and $E \subset\left(\operatorname{Re} z \geq x_{2}\right)$, then $\lambda^{2} \geq \pi\left(x_{2}-x_{1}\right)^{2}$ $\cdot(\operatorname{area} \Omega)^{-1}$. Hence

$$
\omega\left(z_{0}, E, \Omega\right) \leq \exp \left(1-\pi\left(x_{2}-x_{1}\right)^{2}(\operatorname{area} \Omega)^{-1}\right) .
$$

Assume that $\Omega$ is unbounded on the right, and that $f$ is analytic in $\Omega,|f| \leq 1$ on $\partial \Omega$, but $f$ is unbounded in $\Omega$. Comparing $\log |f|$ with the harmonic measure $\omega\left(z, \operatorname{Re} z=x_{2}, \Omega^{\prime}\right)$, where $\Omega^{\prime}=\Omega \cap\left(\operatorname{Re} z<x_{2}\right)$, one obtains for sufficiently large $x_{1}$ and $x_{1}<x_{2}$

$$
\log \log M\left(x_{2}\right) \geq \pi\left(x_{2}-x_{1}\right)^{2}\left(\operatorname{area} \Omega^{\prime}\right)^{-1} .
$$

Denjoy's conjecture, in the strong form (1.1), easily follows.

To prove (3.1), Beurling takes $\Omega$ to be the unit disk $\Delta, z_{0}=0$, and considers mappings $F$ of the disk with

$$
\operatorname{area} F(\Delta)=\int_{\Delta}\left|F^{\prime}(z)\right|^{2} d x d y \leq \pi .
$$

Write $\omega(E)=\omega(0, E, \Delta)$ and define

$$
G(r, \varphi)=\int_{0}^{r}\left|F^{\prime}\left(t e^{i \varphi}\right)\right| d t .
$$

The main estimate is

$$
\omega\left(\left\{e^{i \varphi}: G(1, \varphi)>t\right\}\right) \leq e^{1-t^{2}} .
$$

Given $E \subset \partial \Delta$, take $t^{2}=1-\log \omega(E)$. By (3.4) there must be at least one $e^{i \varphi} \in E$ such that $F$ maps the ray $r e^{i \varphi}$ onto a curve of length $\leq t$. Thus $\lambda^{2}(0, E, \Delta) \leq t^{2}$, which is $(3.1)$. 
Beurling obtains (3.4) by working with a first order differential inequality for the mean value

$$
I(r)=\frac{1}{2 \pi \omega(E)} \int_{E} G^{2}(r, \varphi) d \varphi,
$$

where $E \subset \partial \Delta$.

Inequality (3.4) is valid not just for univalent functions, but for all analytic functions $F$ in $\Delta$ with Dirichlet integral $\leq \pi$. A consequence of (3.4) is that such functions satisfy

$$
\omega\left(\left\{e^{i \varphi}:\left|F\left(e^{i \varphi}\right)-F(0)\right|>t\right\}\right) \leq e^{1-t^{2}},
$$

and hence, for every $\alpha<1$,

$$
\int_{0}^{2 \pi} \exp \left(\alpha\left|F\left(e^{i \varphi}\right)-F(0)\right|^{2}\right) d \varphi \leq C_{\alpha}<\infty .
$$

Recently, Chang and Marshall [CM] obtained the endpoint version of this inequality by proving that (3.5) is still true when $\alpha=1$. Marshall [Mar 2] gives another proof based on an unpublished theorem of Beurling involving extremal length. This theorem is closely related to borderline Sobolev estimates due to Moser and others which play a role in certain problems of geometry. See [Ch] for a survey. Essén $[E]$ has combined Ahlfors's distortion theorem, Tsuji's estimate, and symmetrization techniques to prove a considerable generalization of the ChangMarshall theorem. Finally, we mention that Beurling's inequality is one of the tools in new work by Osgood, Phillips, and Sarnak [OPS] about the spectrum of the Laplacian for plane domains.

Exponential square inequalities have become quite popular lately. Chang, Wilson, and Wolff [CWW] prove a local version of Beurling's theorem by showing that analytic functions in the unit disk with bounded Lusin area function are exponentially square integrable. Bañuelos [Ban] proves a sharper result for functions whose $g^{*}$ function is bounded. The proofs of both of these theorems are based on probability theory. It would be interesting to find classical function theoretic ones, and to settle the open question of whether functions $F$ with bounded Littlewood-Paley $g$-function,

$$
g^{2}(\varphi)=\int_{0}^{1}(1-r)\left|F^{\prime}\left(r e^{i \varphi}\right)\right|^{2} d r
$$

are exponentially square integrable.

Various aspects and generalizations of the Denjoy conjecture have been explored by many authors since 1933 . See, e.g. [J 1]. Here we will take note only of two recent developments. Jenkins [J 2] has given a short proof of a "spiral generalization"which Ahlfors states in his thesis but does not completely prove. The 
other activity is devoted to the question of "small functions". In a note in the 1956 Comptes Rendus, Denjoy returned to his subject of 1907, and conjectured that an entire function of order $\varrho$ can have at most $2 \varrho$ different "asymptotic functions" $b_{i}(z)$ of order $1 / 2$. Denjoy again confirmed his conjecture when the asymptotic paths are rays from the origin. The best known general result at present is due to Fenton $[F]$, who proved that $f$ can have at most $2 \varrho$ asymptotic functions of order $<1 / 4$. By contrast, the analogous small function problem for Nevanlinna's defect relation $\sum \delta(a) \leq 2$ has now been completely settled. See [Dr, §2] for a brief discussion.

\section{The distortion theorem and extremal length}

To prove his distortion theorem Ahlfors makes use of the "length-area method", which [A5, p. 1] he states he learned about in the textbook by Hurwitz and Courant. With the notations of Section 1, set $\omega(x)=u_{2}(x)-u_{1}(x)$. This is the oscillation of $\operatorname{Re} \Phi$ on the crosscut $\theta_{x}$. Ahlfors begins by noting that $\Phi\left(\theta_{x}\right)$ is a curve $\gamma_{x}$ which visits all four sides of an $\omega \times 1$ rectangle, and hence

$$
\int_{\theta_{x}}\left|\Phi^{\prime}(x+i y)\right| d y \geq\left(1+\omega^{2}(x)\right)^{1 / 2} .
$$

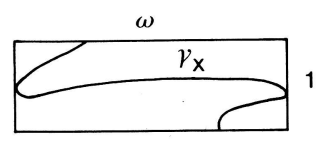

Integration, Schwarz's inequality and the relation

$$
\operatorname{area} \Phi\left(\left\{z \in \Omega: x_{1}<\operatorname{Re} z<x_{2}\right\}\right) \leq u_{2}\left(x_{2}\right)-u_{1}\left(x_{1}\right)
$$

lead to the inequality

$$
\int_{x_{1}}^{x_{2}} \frac{d x}{\theta(x)} \leq u_{1}\left(x_{2}\right)-u_{2}\left(x_{1}\right)+\omega\left(x_{1}\right)+\omega\left(x_{2}\right)-\int_{x_{1}}^{x_{2}} \frac{\omega^{2}(x)}{\theta(x)} d x .
$$

By clever maneuvering with a differential inequality, Ahlfors shows the last three terms on the right can be replaced by 4 , as soon as $\int_{x_{1}}^{x_{2}} d x / \theta(x)>2$.

Ahlfors used variants of the length-area method, and especially integrals of the type $\int d x / \theta(x)$, to prove many other results in the early 1930's. For example, [A 2] contains a " 5 island theorem" for meromorphic functions which generalizes Bloch's theorem about schlicht disks. At about the same time the length-area method was being extensively developed in Germany by Grötzsch, but this work was published in an obscure journal and did not become well known until much later.

A decade later, "presumably in 1943-1944" according to [A 4, p. 81], Beurling further abstracted the ideas in his thesis to conceive the method of extremal length. 
Let $\Gamma$ be any family of rectifiable curves in the plane domain $\Omega$. Its extremal length $\lambda_{\Omega}(\Gamma)$ is defined by

$$
\lambda_{\Omega}(\Gamma)=\sup _{\varrho} \frac{\left(\inf _{\gamma} \int_{\gamma} \varrho(z)|d z|\right)^{2}}{\int_{\Omega} \varrho^{2}(z) d x d y}
$$

where the infimum is taken over all $\gamma \in \Gamma$ and the supremum over all Borel functions $\varrho \geq 0$ on $\Omega$ for which $0<\int_{\Omega} \varrho^{2} d x d y<\infty$. Clearly the extremal length is a conformal invariant: $\lambda_{\Phi(\Omega)}(\Phi(\Gamma))=\lambda_{\Omega}(\Gamma)$ for every conformal mapping $\Phi$.

An introduction to extremal length is given in [A 4], and a more thorough account in [O]. The theory plays a central role in the study of Riemann surfaces and of quasiconformal mappings in the plane and in space. Here we consider only plane domains and will concentrate on one special case, the "extremal distance" between two sets.

For subsets $E_{1}, E_{2}$ of the closure of $\Omega$, the extremal distance $d_{\Omega}\left(E_{1}, E_{2}\right)$ between them is defined to be the extremal length of the family of curves lying in $\Omega$ except for endpoints, one of which is in $E_{1}$, the other in $E_{2}$. For example, it's easy to show that the extremal distance between the $a$-sides of an $a \times b$ rectangle in $b / a$. The "extremal metric", that is , a $\varrho$ for which the supremum is attained in the definition of extremal length, in this case is Euclidean: $\varrho \equiv$ constant.

The theory of extremal length is closely associated with another conformal invariant, the Dirichlet integral. For instance, in the case of extremal distance we have [A 4, p. 65]

$$
d_{\Omega}\left(E_{1}, E_{2}\right)=\left(\int_{\Omega}|\nabla u|^{2}\right)^{-1}
$$

where the function $u$ is harmonic in $\Omega \backslash\left(E_{1} \cup E_{2}\right)$, equals one on $E_{1}$, zero on $E_{2}$, and has vanishing normal derivative on $\partial \Omega \backslash\left(E_{1} \cup E_{2}\right)$. The extremal metric $\varrho$ is given by $\varrho|d z|=|\nabla u||d z|$.

The distance extrémale $\lambda\left(z_{0}, E, \Omega\right)$ of Beurling's thesis, while clearly a forerunner, is not an extremal length in the present sense. The main difference is that in the definition of $\lambda\left(z_{0}, E, \Omega\right)$ only metrics of the form $\varrho(z)|z|=\left|\Phi^{\prime}(z)\right||d z|$, where $\Phi$ is univalent, are permitted in the competition.

To illustrate the workings of the method of extremal length we will outline a conceptually appealing proof of the distortion theorem. For $x_{1}<x_{2}$ let $E_{i}$, $i=1,2$, be the crosscut $\theta x_{i}$, and write $E_{i}^{\prime}=\Phi\left(E_{i}\right)$. Set $\varrho(z)=1 / \theta(x)$ if $z \in \theta_{x}$, $x_{1}<x<x_{2}, \varrho(z)=0$ otherwise. The $\varrho$-length of curves in $\Omega$ joining $E_{1}$ and $E_{2}$ is at least $\int_{x_{1}}^{x_{2}} d x / \theta(x)$, and this integral also equals the $\varrho$-area of $\Omega$. Hence

$$
\int_{x_{1}}^{x_{2}} \frac{d x}{\theta(x)} \leq d_{\Omega}\left(E_{1}, E_{2}\right)=d_{S}\left(E_{1}^{\prime}, E_{2}^{\prime}\right) .
$$

To complete the proof, one must show that the extremal distance $d_{S}\left(E_{1}^{\prime}, E_{2}^{\prime}\right)$ is majorized by the horizontal distance $u_{1}\left(x_{2}\right)-u_{2}\left(x_{1}\right)$, plus a constant. One way 
to do this [A 4, p. 76] is via a reflection and symmetrization argument due to Teichmüller. Jenkins and Oikawa, [JO 2], [J 3], found an even simpler proof. Let $S^{\prime}$ denote the part of $S$ between $E_{1}^{\prime}$ and $E_{2}^{\prime}$, and $F_{1}, F_{2}$ the top and bottom of $S^{\prime}$. Then

$$
d_{S}\left(E_{1}^{\prime}, E_{2}^{\prime}\right)=d_{S^{\prime}}\left(E_{1}^{\prime}, E_{2}^{\prime}\right)=\left(d_{S^{\prime}}\left(F_{1}, F_{2}\right)\right)^{-1} .
$$
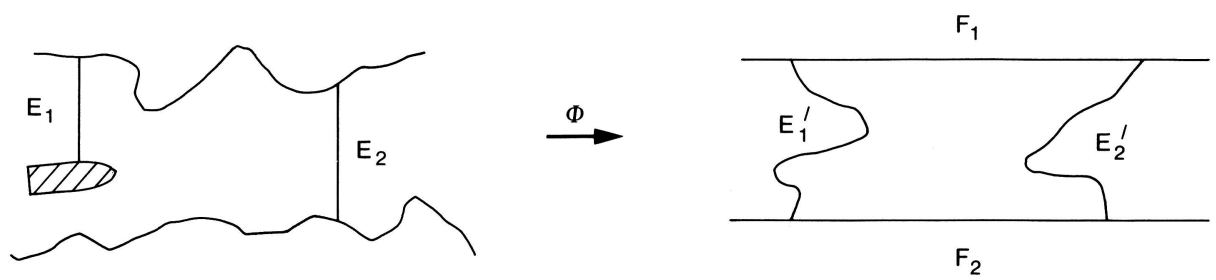

The second equation, the "conjugation principle", is confirmed by mapping $S^{\prime}$ onto a rectangle with $F_{1}, F_{2}$ going to a pair of opposite sides. Jenkins and Oikawa give an explicit choice of $\varrho$ which provides a lower bound for $d_{S^{\prime}}\left(F_{1}, F_{2}\right)$, and the desired upper bound for $d_{S}\left(E_{1}^{\prime}, E_{2}^{\prime}\right)$.

Let $d\left(z_{1}, z_{2}, \Omega\right)$ denote the hyperbolic distance in $\Omega$, normalized as in [A 4] so that $d(0, R)=\log ((1+R) /(1-R))$ when $\Omega$ is the unit disk. The distortion theorem implies the Euclidean-to-conformal inequality

$$
\int_{x_{1}}^{x_{2}} \frac{d x}{\theta(x)} \leq \frac{1}{\pi} d\left(z_{1}, z_{2}, \Omega\right)+4
$$

when the $z_{i}$ are points with $\operatorname{Re} z_{i}=x_{i}$. Thus, if $\Omega$ is narrow the distance is large. In Section 6 we discuss an inequality of this type for harmonic measure.

Theorems of the distortion type play a major role in diverse situations of geometric function theory. For instance, they lie at the base of Hayman's theory of $p$-valent functions [Hay]. Suppose that $f$ is analytic in the unit disk $\Delta$. For $0<R<\infty$ define

$$
p(R)=\frac{1}{2 \pi} \int_{-\pi}^{\pi} n\left(R e^{i \varphi}\right) d \varphi=\frac{1}{2 \pi} \int_{\gamma(R)}\left|f^{\prime}(z)\right||d z|
$$

where $n(w)$ is the number of zeros of $f(z)-w$ in $\Delta$, and $\gamma(R)=\{z \in \Delta:|f(z)|$ $=R\}$. Following up on earlier work of Cartwright and Spencer, Hayman proves an analogue of the distortion theorem [Hay, p. 25],

$$
\int_{R_{1}}^{R_{2}} \frac{d R}{R p(R)} \leq 2 \log \frac{1}{1-r}+A,
$$


where $R_{2}=M(r, f)$, and $R_{1}, A$ are constants which depend on the number of zeros of $f$ and the first few power series coefficients of $f$.

An immediate consequence of (4.1) is that if $f$ takes on each value at most $p$ times then $M(r, f)=O\left((1-r)^{-2 p}\right)$ as $r \rightarrow 1$. Hayman combines (4.1) with Carleman's observation $n^{2} \leq 2 \pi \sum \Theta_{i}^{-1}$ to prove [Hay, p. 32] that a $p$-valent function cannot be too big at too many different places. This theorem, of Denjoy conjecture type, is in turn a key ingredient in Hayman's proof [Hay, p. 104] of the "asymptotic Bieberbach conjecture" that the coefficients $a_{n}$ of a normalized univalent function $f(z)=z+\cdots$ satisfy the relation

$$
\lim _{n \rightarrow \infty} \frac{\left|\alpha_{n}\right|}{n} \leq 1 .
$$

Jenkins and Oikawa [JO 2] give an extremal length proof of (4.1), and, in another paper [JO 1], extend some of Hayman's theorems to a setting involving harmonic functions. See [Ham] for recent work in this direction. We note that (4.1) is a close relative of the theorem of Beurling which Marshall proves in [Mar 2].

In his thesis Ahlfors calls the distortion theorem "die erste Hauptungleichung". He proves also a zweite Hauptungleichung which gives an inequality in the opposite direction, of the form

$$
u_{2}\left(x_{2}\right)-u_{1}\left(x_{1}\right) \leq \int_{x_{1}}^{x_{2}} \frac{d x}{\theta(x)}+K,
$$

provided the domain $\Omega$ satisfies rather strong symmetry and smoothness conditions. It is easy to see that no good result of this sort can be expected for arbitrary $\Omega$, but various authors have been able to substantially weaken Ahlfors's hypotheses. For example, suppose that $\Omega=\left\{z:-\alpha_{1}(x)<y<\alpha_{2}(x),-\infty<x<\infty\right\}$, where $\alpha_{i}>0$. Jenkins and Oikawa [JO 3] prove that (4.2) is true with

$$
K=\left(3 m^{3 / 2}\right)^{-1}\left(V_{1}^{3 / 2}+V_{2}^{3 / 2}\right)+C
$$

where $m=\inf _{x_{1}<x<x_{2}} \min _{1,2} \alpha_{i}(x)$ and $V_{i}$ is variation of $\alpha_{i}$ over $\left[x_{1}, x_{2}\right]$ of order $2 / 3$.

\section{The angular derivative}

Suppose that $F$ conformally maps the right half plane onto an unbounded domain $\Omega$, and that $\lim F(z)=\infty$ when $z \rightarrow \infty$ in any angle $|\arg z| \leq \alpha<\pi / 2$. $\mathrm{F}$ is said to have an angular derivative at $\infty$, or, in the terminology of [JO 4], be conformal at $\infty$, if the limit

$$
\lim _{z \rightarrow \infty} \frac{F(z)}{z}, \quad|\arg z| \leq \alpha<\frac{\pi}{2},
$$


exists and is neither zero nor infinity.

Passing to logarithms in both planes, we can replace the half plane by our strip $S=\{z:|\operatorname{Im} z|<1 / 2\}$ and assume that $F$ maps $S$ onto a striplike $\Omega$. Following [RW 2], we normalize by assuming that $\Omega$ contains the positive real axis, and also that $\lim \operatorname{Re} F(z)=\infty$ when $\operatorname{Re} z \rightarrow+\infty$ in $S$ with $|\operatorname{Im} z| \leq \alpha<1 / 2$. In this formulation, the mapping has an angular derivative if

$$
\lim (F(z)-z)
$$

exists and is finite, with $z$ approaching $\infty$ as just indicated. It is easy to see that this is a property of $\Omega$ rather than $F$. When it holds we shall say that $\Omega$ has an angular derivative (A.D.).

Problem of the angular derivative. Find necessary and sufficient Euclidean geometric conditions on $\Omega$ for it to have an A.D.

This problem is stated by Ahlfors in his thesis [A 1, p. 47]. Loosely speaking, $\Omega$ has an A.D. if it is nearly equal to $S$ at $\infty$, and the question becomes "how nearly".

In [A 1] Ahlfors applied his first and second main inequalities to obtain some necessary and some sufficient conditions for A.D. to exist. For example, if $\Omega_{1}$ has the form

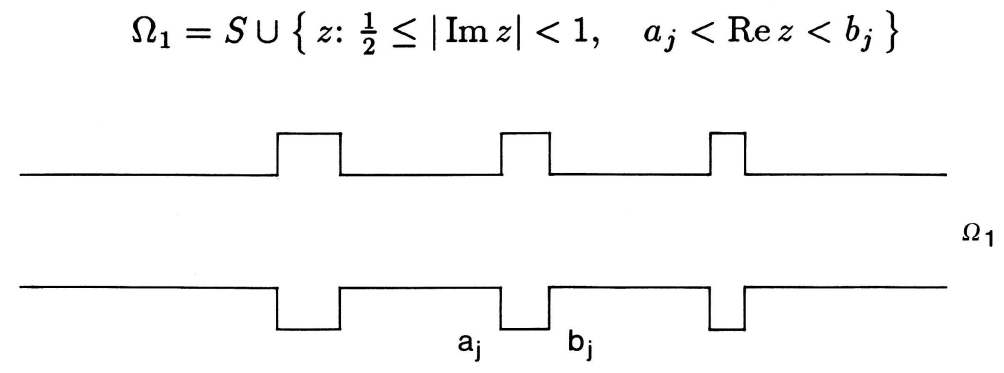

he proved $\sum_{j}\left(b_{j}-a_{j}\right)<\infty$ is sufficient for $\Omega_{1}$ to have an A.D. For domains of the form

$$
\Omega_{2}=S \backslash \bigcup_{j=1}^{\infty}\left(j+i y: \frac{1}{2}-\varepsilon_{j} \leq|y|<\frac{1}{2}\right)
$$

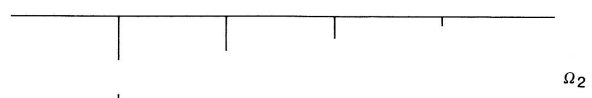

he proved that $\sum \varepsilon_{j}^{2}<\infty$ is necessary and $\sum \varepsilon_{j}<\infty$ is sufficient.

Study of the A.D. has been carried on by many authors. The article [RW 2] will provide a good starting point for the interested reader. 
Jenkins and Oikawa [JO 4] and, independently, Rodin and Warschawski [RW 1], showed that existence of A.D. can be characterized in terms of extremal distance.

Since $\Omega$ contains the positive real

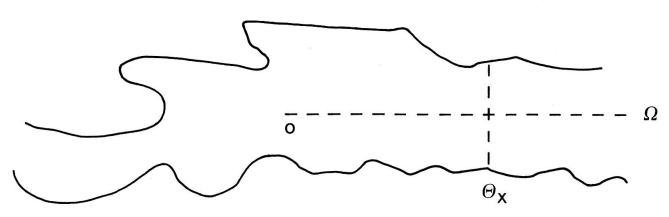
axis there is a crosscut $\theta_{x}$ of $\Omega$ lying on $\operatorname{Re} z=x$ which intersects the real axis. Note that when $\Omega=S$ we have $d_{S}\left(\theta_{x_{1}}, \theta_{x_{2}}\right)=x_{2}-x_{1}$.

Theorem. ([JO 4], [RW 1].) The domain $\Omega$ has an A.D. if and only if, when $x_{1}, x_{2} \rightarrow \infty, x_{1}<x_{2}$,

$$
d_{\Omega}\left(\theta_{x_{1}}, \theta_{x_{2}}\right)=\left(x_{2}-x_{1}\right)+o(1) .
$$

According to [J 3, p. 100] this "must be regarded as its [the A.D. problem's] definitive solution". On the other hand, Rodin and Warschawski [RW 2, p. 1] maintain that the problem of finding a Euclidean characterization remains unsolved, but that the extremal length approach "places the angular derivative problem in the broader context of estimating conformal invariants in terms of Euclidean quantities".

In [RW 2] the authors apply this theorem to give a very explicit geometric solution when either $\Omega \subset S$ or $S \subset \Omega$. If $\Omega \subset S$ then [RW 2, Theorem 1] $\Omega$ has A.D. if and only if the part of $S \backslash \Omega$ in $\operatorname{Re} z>0$ can be covered by disks $\Delta_{n}$ with centers on $\partial S$ and radii $r_{n}$ such that $\sum_{n} r_{n}^{2}<\infty$. If $S \subset \Omega$, say that $\Omega$ contains a square $Q$ which borders $S$ if $Q$ is a square with $Q \subset \Omega$, and either the lower side of $Q$ is contained in $\operatorname{Im} z=1 / 2$ or the upper side in $\operatorname{Im} z=-1 / 2$. The necessary and sufficient condition for $\Omega$ to have an A.D., when $S \subset \Omega$, is that whenever $\Omega$ contains disjoint squares which border $S$, the sum of their areas is finite.

Referring back to the domains $\Omega_{1}$ and $\Omega_{2}$ considered by Ahlfors, it follows that $\Omega_{1}$ has an A.D. if and only if $\sum\left(b_{j}-a_{j}\right)^{2}<\infty$. For $\Omega_{2}$, an A.D. exists if and only if $\sum \varepsilon_{j}^{2}<\infty$.

Burdzy [Bur], using Brownian excursion theory, has recently given a substantial generalization of Theorem 2 of [RW 2]. He assumes that $S \backslash \Omega$ is small, and gives a necessary and sufficient smallness condition on $\Omega \backslash S$ for A.D. to exist. Burdzy phrases his smallness condition in terms of Lipschitz minorants of $\partial \Omega$. In [RW 3] the authors show that Burdzy's theorem can be restated in terms of the covering conditions of [RW 2]. They give also a proof of the sufficiency part based on extremal length.

Carroll [Crl] has given a "classical" proof of the necessity by constructing, when $S \backslash \Omega$ is small but $\Omega \backslash S$ is large, a function $u$ subharmonic in $\Omega, \leq 0$ on $\partial \Omega$, which satisfies

$$
\lim _{x \rightarrow+\infty} e^{\pi x} u(x)=+\infty .
$$


From this, he is able to deduce that the conformal mapping $F: S \rightarrow \Omega$ must satisfy $\lim _{x \rightarrow+\infty}[\operatorname{Re} F(x)-x]=+\infty$.

The domains $\Omega$ for which it is not yet possible to decide quickly the A.D. question on the basis of Euclidean data neither contain nor are contained in $S$. The "comb domains " furnish an intersting class. For $a>0, \varepsilon>0$, let $L(a, \varepsilon)$ denote the union of the vertical rays above $a+i\left(\frac{1}{2}-\varepsilon\right)$ and below $a-i\left(\frac{1}{2}-\varepsilon\right)$. Define

$$
\Omega=\mathbf{C} \backslash \bigcup_{j=1}^{\infty} L\left(a_{j}, \varepsilon_{j}\right) .
$$

If $\sum_{j=1}^{\infty} \varepsilon_{j}^{2}<\infty$, it follows from Burdzy'z theorem that $\sum_{j=1}^{\infty}\left(a_{j+1}-a_{j}\right)^{2}<$ $\infty$ is necessary and sufficient for $\Omega$ to have an A.D. But if, for example, $\varepsilon_{j}=j^{-1 / 2}$, it is apparently not known which range of $a_{j}$ is the right one.

\section{Harmonic measure}

We discussed Tsuji's estimate for harmonic measure in Section 2. Here we shall concentrate on the case of simply connected domains.

Consider first the unit disk $\Delta$, and let $E$ be a closed subset of $\partial \Delta$. Let $\gamma(E)=\log (\operatorname{cap} E)^{-1}$ denote the Robin constant of $E$, where cap is the logarithmic capacity, and $C_{r}$ denote the circle $|z|=r$. In [AB 2], Ahlfors and Beurling prove a relation between capacity and extremal distance:

$$
\gamma(E)=\pi \lim _{r \rightarrow 0}\left[d_{\Delta}\left(C_{r}, E\right)-d_{\Delta}\left(C_{r}, \partial \Delta\right)\right] .
$$

The limit on the right is the "reduced extremal distance" in $\Delta$ from $E$ to the origin. Theorem 4.9 of [A 4] gives a more general result of this type.

Pfluger [Pf] gave a variant of (6.1). Suppose that $K$ is a compact subset of $\Delta$, which we take to be a small disk centered at the origin. Then

$$
\left|\gamma(E)-\pi d_{\Delta}(K, E)\right| \leq C,
$$


where $C$ depends only on $K$.

The proofs of both (6.1) and (6.2) are based on the Dirichlet integral characterization of extremal distance discussed in Section 4.

Since $\operatorname{cap} E \geq \sin \left(\frac{1}{2} \pi \omega(0, E, \Delta)\right)$ ([A 4, p. 35]), Pfluger's theorem implies an inequality between two conformal invariants

$$
\omega\left(z_{0}, E, \Omega\right) \leq C e^{-\pi d_{\Omega}(K, E)},
$$

where $\Omega$ is any simply connected domain, $E \subset \partial \Omega, z_{0} \in \Omega$, and for $K$ we take a closed disk centered at $z_{0}$ whose radius is, say, one half the distance from $z_{0}$ to $\partial \Omega$. The constant $C$ is then absolute.

There are many situations in which one can estimate $d_{\Omega}(K, E)$ from below in terms of Euclidean data, and then (6.3) gives an upper bound for harmonic measure. Consider, for instance, the striplike domain $\Omega$ discussed in connection with Ahlfors's distortion theorem in Sections 1 and 4. Let $z_{0}=x_{1}+i y_{1}, x_{2}>x_{1}$, $\Omega^{\prime}=\Omega \cap\left(\operatorname{Re} z<x_{2}\right)$ and $E=\theta_{x_{2}}$, the crosscut considered there. The distortion theorem provides the required estimate of $d_{\Omega^{\prime}}\left(K, \theta_{x_{2}}\right)$, and from (6.3) we deduce

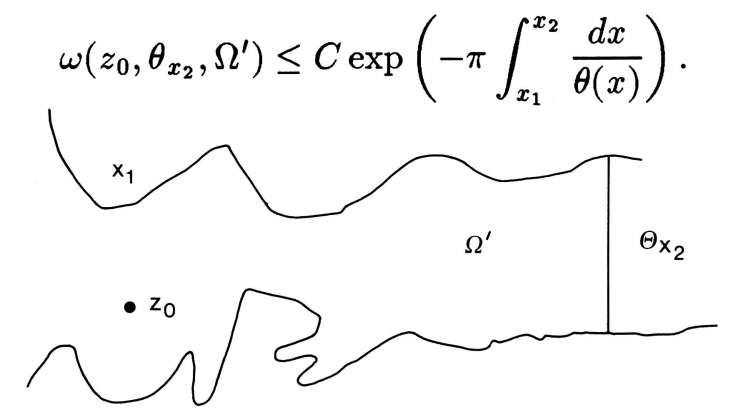

This inequality is especially interesting when $\theta_{x_{2}}$ is all of $\left(\operatorname{Re} z=x_{2}\right) \cap \Omega$. In that case it is better than the one given by logarithmic transformation of Tsuji's inequality (2.2).

Hersch [He] obtains a related result by means of explicit identities connecting extremal length with harmonic measure and hyperbolic distances.

Pfluger's inequality (6.3) is one of the tools in the 1985 work of Makarov [Mak 1], [Mak 2] about relations between harmonic and Hausdorff measures. Suppose that $\Omega$ is a bounded Jordan domain and $E \subset \partial \Omega$. Let $\Lambda_{\alpha}$ denote $\alpha$ dimensional Hausdorff measure, and $\omega$ denote harmonic measure at some fixed point of $\Omega$. From the Beurling-Nevanlinna projection theorem [A 4, p. 43] it is easy to prove that $\Lambda_{1 / 2}(E)=0$ implies $\omega(E)=0$. That is, $\omega<<\Lambda_{1 / 2}$.

Carleson, in 1973, proved via a very complicated argument that $\omega<<\Lambda_{1 / 2+\varepsilon}$ for some absolute $\varepsilon>0$, and conjectured that $\omega<<\Lambda_{\alpha}$ should be true for every $\alpha<1$. Examples going back to Lavrentiev in 1936 show that $\omega<<\Lambda_{1}$ can fail.

Makarov proved Carleson's conjecture in an even stronger form. 
Makarov's theorem. There is an absolute constant $C$ such that $\Lambda_{h}(E)=0$ implies $\omega(E)=0$, where

$$
h(t)=t \exp \left(C\left(\log \frac{1}{t}\left(\log \log \log \frac{1}{t}\right)\right)^{1 / 2}\right) .
$$

This theorem is astonishingly precise. Makarov also shows that for small enough $c$ there are quasidisks for which $\omega$ and the corresponding $\Lambda_{h}$ are mutually singular.

Let $F$ be a conformal mapping of the unit disk $\Delta$ onto $\Omega$. The content of Makarov's theorem is that $F$ cannot be too compressive on $\partial \Delta$. Suppose that $\psi(t)$ is nondecreasing and continuous, $\psi(0)=0$. Let $\chi(t)$ denote the inverse of the function $t \rightarrow t \psi(t)$. One of Makarov's intermediate results is that lower bounds on $\left|F^{\prime}\right|$ in $\Delta$ propagate to lower bounds on $\partial \Delta$. Precisely, if $\Delta_{\varepsilon}$ is a small disk of radius $\varepsilon$, then

$$
\left|F^{\prime}(z)\right| \geq \psi(1-|z|) \quad \text { in } \quad \Delta \text { implies } \omega\left(\Delta_{\varepsilon} \cap \partial \Omega\right) \leq C \chi(\varepsilon) \log \frac{1}{\varepsilon} .
$$

A key stop in the proof is to show that if $\omega\left(\Delta_{\varepsilon} \cap \partial \Omega\right)$ is relatively large, then so is $\omega(\beta)$, for some connected set $\beta \subset \partial \Omega \cap \Delta_{2 \varepsilon}$. To do this, Makarov uses (6.3), along with "Grötzsch's principle" [A 4, Theorem 4-2B] to estimate the extremal distances.

Along with his theorem that the mapping $F$ cannot be too compressive, Makarov proved also that $F$ cannot be too expansive. A particular result is that $\omega$ is singular with respect to $\Lambda_{1+\varepsilon}$ for every $\varepsilon>0$. This confirmed a conjecture of Øksendal in the simply connected case. Jones and Wolff [JW] have proved that in fact $\Lambda_{1+\varepsilon} \perp \omega$ for every plane domain. For $\Omega \subset \mathbf{R}^{n}$ the analogue of $\varnothing$ ksendal's conjecture was that $\omega$ is always supported on a set of dimension $\leq n-1$. Bourgain [Bo] proved it is supported on a set of dimension $\leq n-\varepsilon$ for some $\varepsilon>0$, but Wolff [Wol] has now shown that the full $n-1$ conjecture is false.

Concerning multiply connected plane domains, Carleson [Csn 2] has given examples in which the topological boundary of $\Omega$ has dimension larger than one but the harmonic measure is supported on a set of dimension smaller than one. This contrasts sharply with the simply connected case, where Makarov's theorems show that the harmonic measure always lives on a set of dimension exactly one.

Returning now to simply connected $\Omega$, a problem closely related to Makarov's compression theorem remains unsolved. According to that theorem, $\left|F^{\prime}\right|$ satisfies certain lower bounds, so that the derivatives of conformal mappings $\Phi$ from $\Omega$ onto the disk $\Delta$ should satisfy upper bounds.

Problem. For which $p$ is it always true that

$$
\int_{\Omega} \int\left|\Phi^{\prime}(z)\right|^{p} d x d y<\infty ?
$$


This is clearly the case for $p=2$. We shall restrict our discussion to $p \geq 2$. Slit disks show it can fail for $p=4$. J. Brennan [Br] was led to this question in his study of approximation theory. Adapting Carleson's argument that $\omega<<\Lambda_{1 / 2+\varepsilon}$, Brennan proved the existence of $\varepsilon>0$ for which integrability holds for $p \leq 3+\varepsilon$. He conjectured that integrability should hold for every $p<4$. The best known result, $p<3.39$, is due to Pommerenke [Po 1], [Po 2], who worked with the inverse mapping $F: \Delta \rightarrow \Omega$ and proved non-sharp upper bounds for means

$$
I(r)=\frac{1}{2 \pi} \int_{-\pi}^{\pi}\left|F^{\prime}\left(r e^{i \theta}\right)\right|^{-p} d \theta, \quad p>0 .
$$

The Carleson-Brennan method relies on extremal length. Pommerenke's slick and simple proofs involve the Schwarzian derivative $S_{F}$ and differential inequalities for $I(r)$, in the spirit of Carleman.

There has also been recent work about integrability of $\left|\Phi^{\prime}\right|$ when one integrates over one-dimensional sets. The basic theorem is due to Hayman and Wu [HWu], who confirmed a conjecture of Piranian and Weitsman by proving that for any conformal mapping $\Phi: \Omega \rightarrow \Delta$ and any line or circle $L$,

$$
\int_{\Omega \cap L}\left|\Phi^{\prime}(z)\right||d z| \leq C<\infty .
$$

Simpler proofs have been given by Garnett, Gehring, and Jones [GGJ] and Fernández, Heinonen, and Martio [FHM]. In [HWu] it is shown that $C=10^{35}$ is permissible. In [FHM] this bound is reduced to $C=4 \pi^{2}$ and a conjecture is advanced for the best constant. The papers [GGJ], [FHM], [FZ], and [FH] contain extensions in which lines are replaced by more general curves. The definitive result of this type has now been proved by Bishop and Jones (preprint, Yale University), who prove that (6.5) holds for a curve $L$, with $C=C(L)$, if $L$ is "Ahlfors regular". These curves are defined in [Dr, Section $8 \mathrm{E}]$. The necessity of Ahlfors regularity for (6.5) had been shown in [GGJ].

Returning to the case of lines, one may ask for which $p \geq 1$ is it always true that

$$
\int_{L \cap \Omega}\left|\Phi^{\prime}(z)\right|^{p}|d z|<\infty
$$


In $[\mathrm{FHM}]$ it is proved that $\Phi^{\prime} \in L^{p}(L \cap \Omega)$ for all $p \leq$ $1+\varepsilon$ for some absolute $\varepsilon>0$. The analogue of Brennan's conjecture is that integrability should hold for every $p \in$ $[1,2)$. But this turns out to be false. There is a "tree" $T$, of the type pictured, so that for $\Omega=\mathbf{C} \backslash T$ we have

$$
\int_{\mathbf{R} \cap \Omega}\left|\Phi^{\prime}(x)\right|^{p} d x=\infty
$$

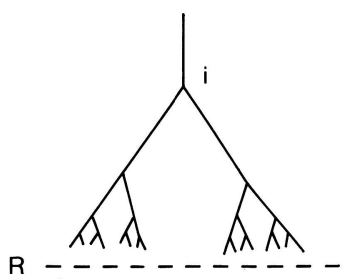

for some $p<2$ [Bae 2].

The theory of harmonic measure advances on many fronts. Marshall and Sundberg [MS] used their computer to guess the extremal case for a problem of Hall's lemma type, and then used extremal length to confirm their guess. Dubinin $[\mathrm{Du}]$, see also [Bae], invented a new tool, "desymmetrization", to solve a "reverse extremal problem" about slit disks, thereby proving a conjecture of Gončar. Lewis and $\mathrm{Wu}[\mathrm{LW}]$ proved some difficult estimates for harmonic measure in multiply connected domains which enabled them to prove a conjecture of Littlewood about the spherical derivative of polynomials and to put a dent in a conjecture of Arakelyan about deficiencies of entire functions.

Various aspects of recent work on harmonic measure are surveyed in [Csn 1] and [G 3]. The older article [Hal] contains much information still of interest.

\section{Analytic capacity}

In 1888 Painlevé took up the following problem: Give Euclidean conditions on a compact set $K$ so that whenever $K$ is contained in a domain $\Omega$ and $f$ is a bounded analytic function on $\Omega \backslash K$, then $f$ has an analytic extension to $\Omega$. No condition which is both necessary and sufficient has yet been found. Such sets will be called Painlevé null. It is not hard to see that $K$ is Painlevé null if and only if the space $H^{\infty}\left(K^{c}\right)$ of bounded holomorphic functions in the complement of $K$ consists only of constants.

In 1947 Ahlfors [A 3] introduced a quantitative version of Painlevé's problem. Define

$$
\gamma(K)=\sup \left\{\left|f^{\prime}(\infty)\right|:\|f\|_{H^{\infty}\left(K^{c}\right)} \leq 1\right\},
$$

where $f^{\prime}(\infty)=\lim _{z \rightarrow \infty} z(f(z)-f(\infty))$. The number $\gamma(K)$ is now known as the analytic capacity of $K$. It is clear that $\gamma(K)=0$ if and only is $K$ is Painlevé-null.

If the one-dimensional Hausdorff measure $\Lambda_{1}(K)$ is zero, then also $\gamma(K)=0$. This result goes back to Painlevé. It is also classical that $\Lambda_{1+\varepsilon}(K)>0$ implies $\gamma(K)>0$. On the other hand, $\Lambda_{1}(K)>0$ does not imply $\gamma(K)>0$. Proofs of these results may be found in [G 1].

In 1950, Ahlfors and Beurling [AB 1] studied Painlevé null sets, along with those for related function classes. This paper contains the first account of extremal 
length to appear in a widely circulated journal. Among their results is a theorem that $K$ is null for the class of analytic functions with finite Dirichlet integral if and only if removal of $K$ does not increase the extremal distance between pairs of sets. In case $K$ is a subset of the unit circle, they prove also that $K$ is Dirichlet-null if and only if the complement of $K$ in the circle has inner log-capacity equal to one. Since capacity is describable in terms of transfinite diameter this qualifies as a Euclidean characterization. But for arbitrary compact sets it appears that, as in the case of Painlevé null sets, such a characterization remains unknown.

Concerning Painlevé's problem, Denjoy in 1909 asserted that if $K$ is a subset of a rectifiable curve $\Gamma$, then $\gamma(K)=0$ if and only if $\Lambda_{1}(K)=0$. Denjoy's proof was valid only in the case when $\Gamma$ is a line. Ahlfors and Beurling [AB] gave a proof of the case when $\Gamma$ is analytic. The truth of Denjoy's assertion for arbitrary rectifiable $\Gamma$ was finally established in 1977, as a consequence of Calderón's theorem about boundedness of the Cauchy integral on curves together with various other machinery such as Garabedian's duality theory. A proof is outlined in [Mar 1], where the reader will find much other interesting information. The proof that $\Lambda_{1}(K)>0$ implies $\gamma(K)>0$ is purely existential. Except in special cases it still is not known how actually to construct bounded analytic functions in the complement of $K$.

In 1967 Vitushkin, see [Mar 1], offered a conjecture for the Euclidean characterization of general Painlevé null sets. Let $\ell_{\theta}$ denote the ray $\arg z=\theta$ and $\left|P_{\theta}(K)\right|$ the Lebesgue measure of the orthogonal projection of $K$ on $\ell_{\theta}$. Define

$$
\mathrm{Bu}(K)=\frac{1}{2 \pi} \int_{0}^{2 \pi}\left|P_{\theta}(K)\right| d \theta .
$$

This expression was introduced by Crofton in 1868 in connection with his solution of the Buffon needle problem. Let $L(r, \theta)$ denote the line passing through $r e^{i \theta}$ and orthogonal to $\ell_{\theta}$. If $K$ is contained in the unit disk, then $\mathrm{Bu}(K)$ represents the probability, measured via $d r d \theta / 2 \pi$, that a randomly chosen "needle" $L(r, \theta)$ will intersect $K$.

Vitushkin's conjecture was that $K$ is Painlevé null if and only if $\mathrm{Bu}(K)=0$. But this turns out to be false. Mattila [Mat] gives an indirect disproof by showing that $\mathrm{Bu}(K)=0$ is not a conformally invariant condition. An explicit disproof has been given by Jones and Murai, who construct a set $K$ with $\gamma(K)>0$ but $\mathrm{Bu}(K)=0$. See also [Mu 1]. Murai [Mu 2] has improved on this by constructing for each $\alpha \in(0,1 / 2)$ a set $K_{\alpha}$ with $\gamma\left(K_{\alpha}\right)>0$ but "Crofton $\alpha$-measure" zero. Murai's lecture notes [Mu 2] provide a rich account of new work on relations between analytic capacity and theorems of Calderón type about boundedness of the Cauchy integral operator on curves. These notes contain also a "simple" proof of the original Calderón theorem due to Jones and Semmes.

As far as I know, no new conjecture concerning Euclidean characterization of Painlevé null sets has been advanced to take the place of Vitushkin's. 
If a domain $\Omega$ does carry some bounded non-constant analytic functions, one can then ask if it carries a lot of them. The "corona problem" provides a specific formulation. Suppose that $f_{1}, \ldots, f_{n} \in H^{\infty}(\Omega)$ and $\inf _{z \in \Omega} \sum_{j}\left|f_{j}(z)\right|>0$. Do there exist $g_{j} \in H^{\infty}(\Omega)$ such that $\sum f_{j} g_{j} \equiv 1$ ? If such $g_{j}$ always exist, we say $\Omega$ has the corona property.

Carleson proved in 1962 that simply connected domains have the corona property. It was proved shortly thereafter that the same is true for finitely connected domains. But Cole, in 1970, showed there are Riemann surfaces for which the corona property does not hold. It is an open question whether every plane domain has the corona property. Garnett's book [G 2] and survey [G 4] provide good introductions to this subject.

In case $K$ is contained in a line Garnett and Jones [GJ] proved that $\Omega=K^{c}$ does have the corona property. This is an analogue of Denjoy's theorem from 1909 that for linear sets $\gamma(K)>0 \leftrightarrow \Lambda_{1}(K)>0$. Moore [Mo] has extended the Garnett-Jones theorem to the case when $K$ is contained in a $C^{1+\varepsilon}$ curve, but the $C^{1}$ case is still unsolved. The proof by Garnett and Jones uses a reflection argument which appears to have no obvious analogue for general curves. Probably the more basic difficulty is our lack of knowledge about how actually to construct $H^{\infty}$ functions in the complement of $K$.

\section{References}

[A 1] AhlFors, L.V.: Untersuchungen zur Theorie der Konformen Abbildung und der ganzen Funktionen. - Acta Soc. Sci. Fenn. (N.S.) 1, 9, 1930, 1-40.

[A 2] Ahlfors, L.V.: Sur les domaines dans lesquels une function méromorphe prend des valeurs appartenant à une région donnée. - Acta. Soc. Sci. Fenn. (N.S.) 2, 2, 1933, $1-17$.

[A 3] Ahlfors, L.V.: Bounded analytic functions. - Duke Math. J. 14, 1947, 1-11.

[A 4] Ahlfors, L.V.: Conformal invariants: Topics in geometric function theory. - McGraw-Hill Book Company, New York, 1973.

[A 5] Ahlfors, L.V.: Collected papers. Volume 1. - Birkhäuser, Boston, 1982.

[AB 1] Ahlfors, L.V., and A. BeuRling: Conformal invariants and function-theoretic null-sets. - Acta Math. 83, 1950, 100-129.

[AB 2] Ahlfors, L.V., and A. Beurling: Conformal invariants. Construction and applications of conformal maps. - National Bureau of Standards, Appl. Math. Ser. 18, 243-45. U.S. Government Printing Office, 1952.

[Bae] Baernstein, A.: Dubinin's symmetrization theorem. - Complex analysis I. Proceedings, University of Maryland 1985-86, (C.A. Berenstein, editor). Lecture Notes in Mathematics 1275. Springer-Verlag, Berlin-Heidelberg, 1987, 23-30.

[Bae 2] BAERnstein, A.: A counterexample concerning integrability of derivatives of conformal mappings. - J. Analyse Math. (to appear).

[Ban] Bañuelos, R.: Brownian motion and area functions. - Indiana Univ. Math. J. 35, 1986, 643-668.

[Be] Beurling, A.: Études sur un problème de majoration. - Thèse, Uppsala, 1933.

[Bo] BourgaIN, J.: On the Hausdorff dimension of harmonic measure in higher dimension. Invent. Math. 87, 1987, 477-483. 
[Br] Brennan, J.: Integrability of the derivative in conformal mapping. - J. London Math. Soc. 18, 1978, 261-272.

[Bur] Burdzy, K.: Brownian excursions and minimal thinness. Part III: Applications to the angular derivative problem. - Math. Z. 192, 1986, 89-107.

[Cmn] Carleman, T.: Sur une inégalité différentielle dans la théorie des fonctions analytiques. - C.R. Acad. Sci. Paris, 196, 1933, 995-997.

[Crl] CARroll, T.: A classical proof of Burdzy's theorem on the angular derivative. - J. London Math. Soc. (to appear).

[Csn 1] Carleson, L.: Estimates of harmonic measures. - Ann. Acad. Sci. Fenn. Ser. A I Math. $7,1982,25-32$.

[Csn 2] Carleson, L.: On the support of harmonic measure for sets of Cantor type. - Ann. Acad. Sci. Fenn. Ser. A I Math. 10, 1985, 113-123.

[Ch] ChANG, S-Y.A.: Extremal functions in a sharp form of Sobolev inequality. - Proceedings of the International Congress of Mathematicians 1986, Volume I. American Mathematical Society, Providence, 1987, 715-723.

[CM] Chang, S-Y.A., and D. Marshall: On a sharp inequality concerning the Dirichlet integral. - Amer. J. Math. 107, 1985, 1015-1033.

[CWW] Chang, S-Y.A., J.M. WILson, and T.H. WolfF: Some weighted norm inequalities concerning the Schrödinger operators. - Comment. Math. Helv. 60, 1985, 217-246.

[Dr] Drasin, D.: The impact of Lars Ahlfors's work in value distribution theory. - Ann. Acad. Sci. Fenn. Ser. A I Math. 13, 1988, - .

[Du] Dubinin, V.N.: On the change of harmonic measure under symmetrization. - Mat. Sb. 124, 1984, 272-279 (Russian). English translation: Math. USSR-Sb. 52, 1985, 267-273.

[E] EsSÉN, M.: Sharp estimates of uniform harmonic majorants in the plane. - Ark. Mat. 25, 1987, 15-28.

[F] Fenton, P.: Entire functions having asymptotic functions. - Bull. Austral. Math. Soc. $27,1983,321-328$.

[FH] Fernández, J., and D. HAmilton: Lengths of curves under conformal mapping. - Comment. Math. Helv. 62, 1987, 122-134.

[FZ] FernándeZ, J., and M. Zinsmeister: Ensembles de niveau des representations conformes. - C.R. Acad. Sci. Paris Ser. I Math. 305, 1987, 449-452.

[FHM] Fernández, J., J. Heinonen, and O. Martio: Quasilines and conformal mappings. J. Analyse Math. (to appear).

[G 1] Garnett, J.: Analytic capacity and measure. - Lecture Notes in Mathematics 297. Springer-Verlag, Berlin-Heidelberg, 1972.

[G 2] Garnett, J.: Bounded analytic functions. - Academic Press, New York, 1981.

[G 3] GarnetT, J.: Applications of harmonic measure. - The University of Arkansas Lecture Notes in the Mathematical Sciences. Volume 8. Wiley-Interscience, New York, 1986.

[G 4] GaRnETT, J.: Corona problems, interpolation problems, and Cauchy-Riemann equations. - Proceedings of the International Congress of Mathematicians 1986, Volume II. American Mathematical Society, Providence, 1987, 917-923.

[GGJ] Garnett, J., F. Gehring, and P. Jones: Conformally invariant length sums. - Indiana Univ. Math. J. 32, 1983, 809-824.

[GJ] Garnett, J., and P. Jones: The corona theorem for Denjoy domains. - Acta Math. 155, $1985,27-40$.

[Hal] Haliste, K.: Estimates of harmonic measures. - Ark. Mat. 6, 1965, 1-31.

[Ham] Hamilton, D.: A sharp form of the Ahlfors distortion theorem, with applications. - Trans. Amer. Math. Soc. 282, 1984, 799-806. 
[Hay] Hayman, W.K.: Multivalent functions. - Cambridge University Press, Cambridge, 1967.

[HWe] Hayman, W.K., and A. Weitsman: On the coefficients and means of functions omitting values. - Math. Proc. Cambridge Philos. Soc. 77, 1975, 119-137.

[HWu] HaYman, W.K., and J-M.G. WU: Level sets of univalent functions. - Comment. Math. Helv. 56, 1981, 366-403.

[He] Hersch, J.: Longeurs extrémales et théorie des fonctions. - Comment. Math. Helv. 29, 1956, 301-337.

[I] IwANIEC, T.: Hilbert transform in the complex plane and area inequalities for certain quadratic differentials. - Michigan Math. J. 34, 1987, 407-434.

[J 1] Jenkins, J.A.: On the Phragmén-Lindelöf theorem, the Denjoy conjecture, and related results. - Mathematical essays dedicated to A.J. Macintyre (H. Shankar, editor), Ohio University Press, Athens, Ohio, 1970, 183-200.

[J 2] JEnkins, J.A.: On Ahlfors' spiral generalization of the Denjoy conjecture. - Indiana Univ. Math. J. 36, 1987, 41-44.

[J 3] JENkins, J.A.: The method of the extremal metric. - The Bieberbach conjecture (A. Baernstein et al., editors), Mathematical Surveys and Monographs 21. American Mathematical Society, Providence, 1986, 95-104.

[JO 1] JENkins, J.A., and K. OIKaWA: On the growth of slowly increasing unbounded harmonic functions. - Acta Math. 124, 1970, 37-61.

[JO 2] Jenkins, J.A., and K. Oikawa: On results of Ahlfors and Hayman.- Illinois J. Math. 15, $1971,664-671$.

[JO 3] Jenkins, J.A., and K. Oikawa: On Ahlfors' "second fundamental inequality". - Proc. Amer. Math. Soc. 62, 1977, 266-270.

[JO 4] Jenkins, J.A., and K. OikaWa: Conformality and semiconformality at the boundary. J. Reine Angew. Math. 291, 1977, 92-117.

[JW] Jones, P., and T. WolfF: Hausdorff dimension of harmonic measures in the plane. Acta Math. (to appear).

[LW] LEWIS, J.L., and J-M.G. WU: On conjectures of Arakelyan and Littlewood. - J. Analyse Math. (to appear).

[Mak 1] Makarov, N.G.: Distortion of boundary sets under conformal mappings. - Proc. London Math. Soc. (3) 51, 1985, 369-384.

[Mak 2] Makarov, N.G.: Metric properties of harmonic measure. - Proceedings of the International Congress of Mathematicians 1986, Volume I. American Mathematical Society, Providence, 1987, 766-776.

[Mar 1] Marshall, D.: Removable sets for bounded analytic functions. - Linear and complex analysis problem book (V.P. Havin et al., editors), Lecture Notes in Mathematics 1043. Springer-Verlag, Berlin-Heidelberg, 1984, 485-490.

[Mar 2] Marshall, D.: A new proof of a sharp inequality concerning the Dirichlet integral. - Ark. Mat. (to appear).

[MS] Marshall, D., and C. Sundberg: Harmonic measure and radial projection. - Preprint, University of Washington, 1988.

[Mat] Mattila, P.: Smooth maps, null-sets for integralgeometric measure and analytic capacity. - Ann. of Math. 123, 1986, 303-309.

[Mi] Minda, D.: Inequalities for the hyperbolic metric and applications to geometric function theory. - Complex analysis I (C.A. Berenstein, editor), Lecture Notes in Mathematics 1275. Springer-Verlag, Berlin-Heidelberg, 1987, 235-252.

[Mo] Moore, C.: The corona theorem for domains whose boundary lies in a smooth curve. Proc. Amer. Math. Soc. 100, 1987, 266-270. 
[Mu 1] MUraI, T.: Comparison between analytic capacity and the Buffon needle probability. Trans. Amer. Math. Soc. 304, 1987,501-514.

[Mu 2] MuraI, T.: A real variable method for the Cauchy transform and applications to analytic capacity. - Lecture Notes in Mathematics 1307. Springer-Verlag, Berlin-Heidelberg, 1988.

[O] Ohtsuka, M.: Dirichlet problem, extremal length, and prime ends. - Van Nostrand, New York, 1970.

[OPS] Osgood, B., R. Phillips, and P. SarnaK: Extremals of determinants of Laplacians.J. Funct. Anal. 80, 1988, 148-211.

[Pf] Pfluger, A.: Extremallängen und Kapazität. - Comment. Math. Helv. 29, 1955, 120-131.

[Po 1] Pommerenke, Ch.: On the integral means of the derivative of a univalent function. - J. London Math. Soc. 32, 1985, 254-258.

[Po 2] Pommerenke, Ch.: The growth of the derivative of a univalent function. - The Bieberbach conjecture (A. Baernstein et al., editors). American Mathematical Society, Providence, 1986, 143-152.

[RW 1] Rodin, B., and S. WARSChawSKI: Extremal length and the boundary behavior of conformal mappings. - Ann. Acad. Sci. Fenn. Ser. A I Math. 2, 1976, 467-500.

[RW 2] Rodin, B., and S. WARSchaWski: Extremal length and univalent functions, I. The angular derivative. - Math. Z. 153, 1977, 1-17.

[RW 3] Rodin, B., and S. WarschaWski: Remarks on a paper of K. Burdzy. - J. Analyse Math. $46,1986,251-260$.

[R] Rossi, J.: Second order differential equations with transcendental coefficients. - Proc. Amer. Math. Soc. 97, 1986, 61-66.

[S] Shen, L-C.: Proof of a conjecture of Bank and Laine regarding the product of two linearly independent solutions of $y^{\prime \prime}+A y=0$. - Preprint, Syracuse University.

[T] TsujI, M.: Potential theory in modern function theory. - Maruzen Co., Ltd., Tokyo, 1959.

[We 1] Weitsman, A.: A theorem on Nevanlinna deficiencies. - Acta Math. 128, 1972, 41-52.

[We 2] Weitsman, A.: Symmetrization and the Poincaré metric. - Ann. of Math. 124, 1986, 159-169.

[Wo] WolfF, T.: Counterexamples with harmonic gradient in $\mathbf{R}^{3}$. - Preprint, Courant Institute.

Washington University

Department of Mathematics

St. Louis, MO 63130

U.S.A.

Received 18 April 1988 